\title{
Maternal clinical predictors of preterm birth in twin pregnancies: A systemic review involving 2930958 twin pregnancies
}

Shemoon Marleen, MRCOG, ${ }^{1,2,9}$ Chalitha Dimuth MBBS, ${ }^{3}$ Ranmalie Dandeniya MBBS, ${ }^{4}$ Rebecca Macgreggor, MBBS, ${ }^{5}$ Joseph Aquilina, FRCOG, ${ }^{5}$ Asma Khalil, MRCOG ${ }^{6,7}$ Shakila Thangaratinam, $\mathrm{PhD}{ }^{1,8,9}$

\author{
Affiliation: \\ ${ }^{1}$ Barts Research Centre for Women's Health (BARC), Barts and the London School of \\ Medicine and Dentistry, Queen Mary University of London, London, UK. \\ ${ }^{2}$ Sri Jayewardenepura Postgraduate Teaching Hospital, Nugegoda, Sri Lanka. \\ ${ }^{3}$ Department of Obstetrics and Gynaecology, Faculty of Medicine, University of Colombo, \\ Sri Lanka \\ ${ }^{4}$ Department of Obstetrics and Gynaecology, Faculty of Medicine, University of Ruhuna, \\ Sri Lanka \\ ${ }^{5}$ Royal London Hospital, Barts Health NHS Trust, London, UK. \\ ${ }^{6}$ St George's University Hospitals NHS Foundation Trust, London, UK. \\ ${ }^{7}$ St George's Medical School, University of London, UK. \\ ${ }^{8}$ Multidisciplinary Evidence Synthesis Hub (mEsh), Queen Mary University of London, \\ London, UK. \\ ${ }^{9}$ World Health Organization (WHO) Collaborating Centre
}

\section{Author for correspondence:}

Shemoon Marleen MBBS, MD, MRCOG

Barts Research Centre for Women's Health (BARC)

Barts and the London School of Medicine and Dentistry

Queen Mary University of London, London, UK

Email: fathimamarleen@qmul.ac.uk 


\section{Summary}

\section{Background:}

In twin pregnancies, which are at high risk of preterm birth, whether maternal clinical characteristics pose additional risks is not known.

\section{Methods:}

We undertook a systematic review to assess the risk of both spontaneous and iatrogenic early ( $<34$ weeks) or late preterm birth $(<37$ weeks $)$ in twin pregnancies based on maternal clinical predictors. We searched the electronic databases from 1990 to xx 2017 without language restrictions. We included studies on women with monochorionic or dichorionic twin pregnancies that evaluated clinical predictors and preterm births. We reported our findings as odds ratio (OR) with $95 \%$ confidence intervals $(\mathrm{CI})$, and pooled the estimates using random effects meta-analysis for various predictor thresholds. .

\section{Findings:}

From 12, 473 citations, we included 59 studies (2 930958 pregnancies). The risks of early preterm birth were significantly increased in women with twin pregnancies, and had a previous history of preterm birth $\left(\mathrm{OR} 2.67,95 \%\right.$ CI $\left.2.16-3.29, \mathrm{I}^{2}=0 \%\right)$, teenage mothers $(\mathrm{OR}$ $1.81,95 \%$ CI $\left.1.68-1.95, \mathrm{I}^{2}=0 \%\right)$, obese women (OR 1.63, 95\% CI 1.30-2.05, $\left.\mathrm{I}^{2}=52 \%\right)$, nulliparous mothers (OR 1.51, 95\% CI 1.38-1.65, I2=73\%), non-white vs. white (OR 1.31, 95\% CI 1.20-1.43, I2=0\%) mothers, women with diabetes (OR 1.73, 95\% CI 1.29-2.33, I2= $0 \%$ ), and smokers (OR 1.30, 95\% CI 1.23-1.37, I2=0\%). The odds of late preterm birth were also increased in women with history of preterm birth (OR 3.08, 95\% CI 2.10-4.51, I2= $73 \%)$, teenagers $(\mathrm{OR} 1.36,95 \%$ CI 1.18-1.57, I2= 57\%), obese women $(\mathrm{OR} 1.18,95 \% \mathrm{CI}$ $1.02-1.35, \mathrm{I} 2=46 \%$ ), nulliparous (OR $1.41,95 \%$ CI 1.23-1.62, I2= 68\%) and women with hypertension in pregnancy. (OR 1.49, CI 1.20-1.86, I2= 52\%). 


\section{Interpretation:}

Risk factors based on maternal clinical characteristics significantly increase the risks of early and late preterm birth in women with twin pregnancies.

\section{Funding:}

None received.

Keywords:

Word count: 


\section{Introduction}

Preterm birth is the leading cause of perinatal morbidity and mortality in developed countries, accounting for two-thirds of deaths in infants without congenital anomalies. $(1,2)$ The increased incidence of twin pregnancies over the last 30 years has contributed to a further increase in the rates of preterm birth. $(3,4)$ More than half of all twins are born before 37 weeks gestation and a third before 35 weeks.(5) Preterm born twins often need significant support in the short and long term, from complications including neurodevelopmental problems (ref).

Numerous studies and prediction models have been developed to predict the risk of preterm

birth in singletons. ${ }^{(6-11)}$ But this is not the case in twin pregnancies, where only the number of fetuses in a pregnancy is considered as a risk factor for preterm birth. In addition to the type of pregnancy, the added risks from other factors are essential to determine the place and frequency of monitoring, initiation of preventative treatment, timely administration of antenatal corticosteroids. (ref). This is particularly relevant for countries with limited tertiary neonatal care, where high-risk twin pregnancies can benefit from appropriate counselling, early referral and in utero transfer.

The first antenatal visit offers an ideal opportunity to determine the risk of preterm birth in women based on their clinical characteristics. Existing individual studies vary in the risk estimates that are imprecise, as they are often limited by small sample sizes to make robust conclusions. No systematic reviews exist. We undertook a systematic review to assess the risk of early and late preterm birth in twin pregnancies for various maternal characteristics. 


\section{Methods}

We undertook our review using a prospective protocol and complied with reporting guidelines.(12) The PROSPERO ID of this Systematic Review's protocol is CRD42015026465.

Search strategy and study selection criteria

We searched the electronic databases MEDLINE, EMBASE, CINAHL, LILACS (1 January 1990 - November 11, 2017), to identify potentially eligible citations for studies on preterm birth in twin pregnancies, without language restrictions. We used the search terms 'twin pregnancy', 'multiple pregnancies' and combined with terms for outcomes such as 'preterm', 'prematurity', 'preterm birth' or 'premature birth'. We additionally included terms for individual clinical, biochemical and ultrasound predictors and combined with the above terms. We "exploded" the search terms where applicable. The full search strategy is provided in Appendix 1. The electronic search was supplemented with a manual search of the reference lists of all primary studies and previously published systematic reviews.

We selected the studies using a two-stage process. We first identified the relevant citations and then retrieved the full text of the potentially eligible studies. Two independent reviewers undertook study selection (SM and RD/CD). Consensus resolved any disagreements after discussion with a different reviewer (ST). We included studies on women with monochorionic or dichorionic twin pregnancies, which evaluated clinical predictors and preterm births in twin pregnancy. The following maternal clinical predictors were assessed: age, body mass index (BMI), race, parity, history of smoking, previous history of preterm birth, pre-existing or new onset conditions such as diabetes mellitus, anaemia and hypertensive disorders of pregnancy. We included studies if they assessed early preterm birth 
( $<34$ weeks) or late preterm birth $(<37$ weeks), which included spontaneous and iatrogenic preterm delivery. We excluded studies that only reported assisted reproduction related predictors, case reports, case series, in-vitro studies, and animal studies.

Study quality assessment and data extraction

Methodological quality of the included studies was assessed by two independent reviewers (SM and RD/CD) using the Newcastle Ottawa Scale (NOS).(13) We evaluated the risk of bias in the selection, comparability and outcome assessment of cohorts, and allocated stars for adherence to the pre-specified criteria. Studies that scored four stars for selection, two stars for comparability between the cohorts, and three stars for the ascertainment of outcome were regarded to have a low risk of bias. Studies that had two or three stars for selection, one for comparability and two for outcome ascertainment were considered to have a medium risk of bias. Any study with a score of one for selection or outcome ascertainment, or zero for any of the three domains was considered to have a high risk of bias.(14)

Two reviewers (SM and $\mathrm{RD} / \mathrm{CD}$ ) undertook data extraction in duplicate and recorded on a customised data extraction form. Dichotomous data were extracted as $2 \times 2$ tables. We contacted authors of potentially eligible manuscripts by email for relevant data. If multiple studies were published for the same outcomes from the same cohort of subjects, only the most recent study was included.

\section{Statistical analysis}

We pooled the estimates of the individual studies using random effects meta-analysis, and reported the summary estimates as odds ratios (OR) with $95 \%$ confidence intervals (CI) for variously reported thresholds of the predictors. Heterogeneity was assessed with the $\mathrm{I}^{2}$ 
statistic. Publication bias was assessed with funnel plots by plotting the natural logarithm of the ORs against the inverse of the standard error. We statistically checked for the asymmetry of the funnel plot by using Egger's method. (15) Sensitivity analysis was performed by only including women with spontaneous preterm birth and for studies that only included dichorionic twins. Analyses were performed using Revman statistical software(16)

\section{Role of the funding source}

There was no funding source for this study

\section{Results}

From 12, 473 citations, we included 59 studies (2,930,958 pregnancies). Detailed study selection process is shown in figure 1.

Characteristics of the included studies

Of the 59 studies, 15 were prospective cohorts, (17)) 40 were retrospective cohorts (reference), three cohorts were nested within randomised trials (reference), and one was a case-control study(ref). Registry data provided information in 17 studies (reference). 57 studies were conducted in high-income countries (USA 35; Israel 4, Canada 3, Italy 3, UK 3, Sweden 2, Denmark 2, Japan 2, Belgium 1, Korea 1, Brazil 1) while only two studues were conducted in middle-income countries (Iran, South Africa). Most of the studies (88\%, 52/59) were published after 2000. The sample sizes ranged from 20 (Bergelin, 2003) to 779,387 (Vintzileos, 2003).

Half of the included studies $(49 \%, 29 / 59)$ explicitly reported exclusion of complicated twin pregnancies such as major fetal anomalies (20 studies), twin to twin transfusion syndrome (9 
studies), and stillbirth (10 studies). One study excluded (insert reference) monochorionic and eight excluded monoamniotic twin pregnancies (add reference). Five excluded (insert reference) chromosomal abnormalities three (insert reference) excluded selective fetal reduction and one study (insert reference) excluded selective intrauterine growth restriction. Parity was the most commonly reported predictor in almost half of all studies $(47 \%, 28 / 59)$. Over $80 \%$ studies $(85 \%, 50 / 59)$ included an unselected population with women who were both symptomatic and asymptomatic for preterm labour. Treatment of preterm labour or prophylaxis for preterm labour was evaluated in seven studies (insert reference). Three quarters of studies reported on early preterm birth $(75 \%, 44 / 59)$, and on on late preterm birth (76\%, 45/49). A third of all studies reported on spontaneous preterm birth $(32 \%, 19 / 59)$. Table 1 gives the details of the characteristics of the included studies.

Quality of the included studies

Over $75 \%$ (46/59) (insert reference) of the studies were low risk for study selection, 39\% (23/59) were for comparability of cohorts (insert reference), and 93\% (55/59) were for outcome assessment. (insert reference) Medium risk of bias was seen in 22\% (13/59) of studies for study selection (insert reference), 30\% (18/59) for comparability of cohorts (insert ${ }^{\mathrm{i}}$ reference) and 5\% (3/59) for outcome. (insert reference) None of the studies were at high risk of bias for study selection. A third studies were considered to be at high risk for comparability of cohorts $(30 \%, 18 / 59)$ (insert reference) and one study was identified to have high risk of bias for outcome assessment. (insert reference). Quality of included studies shown in figure 2 


\section{Risk factors for preterm birth in twin pregnancies}

Maternal demographic characteristics

Age was reported as a predictor in 15 studies (386,421 pregnancies). In women who were less than 20 years of age, the odds of both early (OR 1.81, 95\% CI 1.68-1.95, I2=0\%), and late preterm birth (OR 1.36, 95\% CI 1.18-1.57, I2= 57\%) were significantly increased. The odds of early preterm birth were significantly reduced in older women above 35 years of age (OR 0.89, 95\% CI (0.82-0.96), I2=37\%) (8 studies, 220,591 pregnancies). There were no significant associations for early or late preterm birth at a threshold of 40 years.

Thirteen studies $(1,468,584$ pregnancies $)$ assessed the relationship between maternal race and twin preterm birth. There was a significant increase in early preterm delivery among nonwhite women compared to white women (OR $1.3195 \%$ CI $(1.20-1.43), \mathrm{I} 2=0 \%)$, and for Black vs. White women (xx). No significant relationship was found between nonwhite women and late preterm birth. Also, no significant associations were identified for both early and late preterm delivery among black women and non-black women.

The relationship between maternal BMI and twin preterm birth was assessed in 10 studies (43982 pregnancies). Obese women with BMI $>35 \mathrm{~kg} / \mathrm{m}^{2}$ ) were at high risk of both early $(\mathrm{OR} 1.63,95 \% \mathrm{CI}(1.30-2.05), \mathrm{I} 2=52 \%)$ and late preterm birth was identified $(\mathrm{OR} 1.18,95 \%$ CI (1.02-1.35), I2=46\%). There were no significant associations both for early and late preterm births in for BMI thresholds $>30 \mathrm{~kg} / \mathrm{m} 2$ and $<19.8$ or $18.5 \mathrm{~kg} / \mathrm{m} 2$.

The relationship between smoking and twin preterm birth was assessed in 15 studies, involving 83,955 pregnancies. In women who smoke, the odds of early preterm birth was 
found to be significantly increased. (OR $1.30,95 \%$ CI $1.23-1.37, \mathrm{I} 2=0 \%$ ) than non-smokers. No differences were observed for late preterm birth.

\section{Pregnancy characteristics}

The relationship between nulliparity and twin preterm birth was assessed in 28 studies (508,021 pregnancies). Nulliparous women were at high risk of both early (OR 1.51, 95\% CI $1.38-1.65, \mathrm{I} 2=73 \%)$ and late preterm birth (OR $1.41,95 \%$ CI $1.23-1.62, \mathrm{I} 2=68 \%)$ than multiparous women.

Nineteen studies (9924 pregnancies) assessed previous preterm delivery as a risk factor. A previous preterm birth was a significant risk factor for both early (OR 2.67, 95\% CI 2.16$3.29, \mathrm{I} 2=0 \%)$ and late preterm birth $(\mathrm{OR} 3.08,95 \% \mathrm{CI} 2.10-4.51, \mathrm{I} 2=73 \%)$ in subsequent pregnancies.

\section{Medical disorders in pregnancy}

The association of maternal diabetes with preterm birth as assessed in 5 studies $(425,918$ pregnancies). Women with diabetes in pregnancy were 1.7 fold more likely have early preterm birth $(\mathrm{OR} 1.73,95 \% \mathrm{Cl} 1.29-2.33, \mathrm{I} 2=0 \%)$ than those without the consition. Hypertensive disorders were assessed in 5 studies involving 281, 376 pregnancies. A significant higher odds of late preterm birth was identified in women with hypertension in pregnancy. (OR 1.49, 95\% Cl (1.20-1.86), I2=52\%). No significant association was found for early preterm birth.

No statistically significant relationship was found between maternal anaemia in twin pregnancy for both early and later preterm birth. ( One study involving 80495 pregnancies). 
Sensitivity analysis

- $\quad$ please give results of spontanous PTB - providing estimates of positive findings

- give results of dichorionic alone studies

Publication bias

\section{Discussion}

This systematic review has provided precise quantitative estimates of the association between maternal clinical predictors and preterm delivery in twin pregnancies. In addition to the underlying risk of prematuraity due to being pregnant with twins, being young (age $<20$ years), obese (BMI > $35 \mathrm{~kg} / \mathrm{m} 2)$, nulliparous, or multiparous with a previous history of preterm delivery is associted with an increased risk of both early and late preterm birth. For early preterm birth where iatrogenic preterm delivery is less likely, additonal risk factors such as non-white race, smoking and diabetes in pregnancy were found to be associated with a significant increase risk of early preterm birth. This information will help in the counselling of women very early in their pregnancy regarding their increased risk of preterm delivery, facilitating early referral, closer surveillance and preventive treatment.

To our knowledge, our review is the first comprehensive assessment of the association between maternal clinical preditors and preterm birth in twin pregnancies. We performed a detailed literature search without language restrictions, thereby increasing our potential to capture all relevant studies. The review was done with a prospective protocol, and we explored the sources of heterogeneity. We assessed study quality in detail and the effect of study quality on the results. Due to the large sample size in this metaanalysis we could provide results with high precision for important clinical predictors. 
The studies varied in characteristics of the population; the exclusion citeria such as monochionic pregnancies and its complications, fetal structural and chromosomal anomalies, seletive fetal reduction, selective intrauterine growth restriction and still birth; selection of women who were either symptomatic for pretrem labour, asymptomatic or both; treatment of preterm labour; type of treatment of preterm labour and outcomes such as gestation of preterm birth and type of preterm birth. Fewer studies were published on maternal clinical predictors and spontenous preterm delivery pregnancy, which contributed to a reduced precision in the findings for this group. Also, due to paucity of data we were unable to assess the outcomes for dichorionic twins as a subgroup.

From the results of this review the clinical predctors that are important to singletons appears to be applicable for twin pregnancies. A history of previous preterm delivery appears to be the strongest predictor of preterm birth in twin pregnancies both for early and late preterm births including spontanous preterm birth. This was supported by the systematic review done by Kazemier et al in 2014 where a previous singleton preterm delivery had 57\% (CI 51.9\%$61.9 \%$ ) absolute risk of recurence of spontanous birth before 37 weeks in twin pregnancies.

However, what appeaars to be conflicting is that age greater than 35 years appears to be a protective factor for preterm birth amoung twin pregnancies which is not in keeping with what is known for singleon pregnancies. Amoung the clinical predictors this was the only predictor found to be a protective for preterm deliveries in twin pregnancies. This finding could be due older women being more likely to be multiparous. 
Maternal anaemia did not show significant asssociation with preterm twin delivery. However this finding may have been due to lack of studies asessing the association between maternal anaemia and twin pretem delivery.

All guidelines have consistently emphasised the absence of robust evidence in predicting preterm birth amoung twin pregnancies. Much of the focus on predictimg preterm birth in twin pregnancies has been on cervical length assessment and fetal fibronectin. Current evidence for predicting preterm birth based on existing systematic reviews and IPD metanaysis supports the use of cervical length screening at 18 weeks for asymptomatic twin pregnancies. Fetal fibronectin demonstrates limited predictive accuracy in aymptomatic twin pregnancy and is currently not recommmded by NICE for predicting preterm birth in twin pregnancies. However, not all units have access to ultrasound scans and fetal fibronectin more so in developing countries with limited resources. Maternal clinical preditors therefore play an important role in identifiying twin pregnancies at high risk of preterm birth.

\section{References}

1. Goldenberg RL. The management of preterm labor. Obstet Gynecol. 2002;100(5 Pt 1):102037.

2. Algwiser A, Al Sultan S, Mesleh RA, Ayoub H. Twin pregnancies: Incidence and outcome Riyadh Armed Forces Hospital experience. Journal of Obstetrics and Gynaecology. 1999;19(6):58793.

3. ACOG Practice Bulletin \#56: Multiple gestation: complicated twin, triplet, and high-order multifetal pregnancy. Obstet Gynecol. 2004;104(4):869-83.

4. Vachharajani AJ, Vachharajani NA, Dawson JG. Comparison of short-term outcomes of late preterm singletons and multiple births: an institutional experience. Clin Pediatr (Phila). 2009;48(9):922-5.

5. Goldenberg RL, lams JD, Miodovnik M, Van Dorsten JP, Thurnau G, Bottoms S, et al. The preterm prediction study: risk factors in twin gestations. National Institute of Child Health and Human Development Maternal-Fetal Medicine Units Network. American Journal of Obstetrics \& Gynecology. 1996;175(4 Pt 1):1047-53.

6. Yawn BP, Thompson LR, Lupo VR, Googins MK, Yawn RA. Prenatal drug use in Minneapolis-St Paul, Minn. A 4-year trend. Archives of family medicine. 1994;3(6):520-7. 
7. Cooperstock MS, Bakewell J, Herman A, Schramm WF. Association of sociodemographic variables with risk for very preterm birth in twins. Obstetrics and Gynecology. 1998;92(1):53-6. 8. Honest H, Bachmann L, Ngai C, Gupta J, Kleijnen J, Khan K. The accuracy of maternal anthropometry measurements as predictor for spontaneous preterm birth: a systematic review. 2005.

9. Michaluk A, Dionne MD, Gazdovich S, Leduc L. Prediction of preterm twin birth based on previous premature singleton delivery. American Journal of Obstetrics and Gynecology. 2011;204 (1 SUPPL.):S55.

10. lams JD, Newman RB, Thom EA, Goldenberg RL, Mueller-Heubach E, Moawad A, et al. Frequency of uterine contractions and the risk of spontaneous preterm delivery. $\mathrm{N}$ Engl J Med. 2002;346(4):250-5.

11. Roberts WE, Morrison JC. Has the use of home monitors, fetal fibronectin, and measurement of cervical length helped predict labor and/or prevent preterm delivery in twins? Clinical Obstetrics and Gynecology. 1998;41(1):95-102.

12. Preferred Reporting Items for Systematic Reviews and Meta-Analyses: The PRISMA Statement. 2017.

13. Wells G, Shea B, O'Connell D, Peterson j, Welch V, Losos M, et al. The Newcastle-Ottawa Scale (NOS) for Assessing the Quality of non-randomised Studies in Meta-analysis. Proceedings or the Third Symposium on Systematic Reviews beyond the Basics SBOD Improving Quality and Impact. 2000.

14. Viale L, Allotey J, Cheong-See F, Arroyo-Manzano D, McCorry D, Bagary M, et al. Epilepsy in pregnancy and reproductive outcomes: a systematic review and meta-analysis. Lancet. 2015;386(10006):1845-52.

15. Matthias E, Davey SG, Christoph M. Bias in meta analysis detected by a simple, graphical test. British Medical Journal; 1997. p. 629.

16. 5.3. RMRmcpV. Review Manager (Rev man) [computer program] Version 5.3. . Copenhagen: The Nordic Cochrane Centre, The Cochrane Collaboration; 2014.

17. Yang JH, Kuhlman K, Daly S, Berghella V. Prediction of preterm birth by second trimester cervical sonography in twin pregnancies. Ultrasound in Obstetrics and Gynecology. 2000;15(4):28891. 